\title{
Manejo da coroidite interna punctata complicada por membrana neovascular sub-retiniana
}

\author{
Management of punctate inner choroidopathy complicated \\ by subretinal neovascular membrane
}

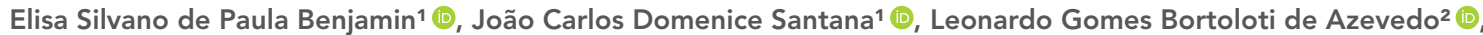

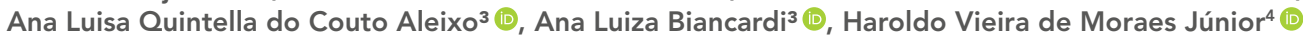

\footnotetext{
'Programa de Residência em Oftalmologia, Hospital Universitário Clementino Fraga Filho, Universidade Federal do Rio de Janeiro, Rio de Janeiro, RJ, Brasil. 2 Programa de Pós-Graduação em Oftalmologia, Setor de Uveítes, Universidade Federal do Rio de Janeiro, Rio de Janeiro, RJ, Brasil. ${ }^{3}$ Fundação Oswaldo Cruz, Rio de Janeiro, RJ, Brasi ${ }^{4}$ Setor de Uveítes, Universidade Federal do Rio de Janeiro, Rio de Janeiro, RJ, Brasil.
}

Descritores:

Coroidite; Neovascularização de coroide; Injeções intravítreas

Keywords:

Choroiditis; Choroidal neovascularization; Intravitreal injections

Recebido: $4 / 5 / 2020$

Aceito:

$5 / 5 / 2020$

Autor correspondente: Elisa Silvano de Paula Benjamin Departamento de Oftalmologia Rua Professor Rodolpho Paulo Rocco, 255 - Ilha do Fundão CEP: 21941-590 - Rio de Janeiro, RJ, Brasil Tel.: (21) 3938-2285

E-mail: elisasilvano@gmail.com

Instituição de realização do trabalho: Universidade Federal do Rio de Janeiro.

Fonte de auxílio à pesquisa: não financiado.

Conflitos de interesse: os autores declaram que não há conflitos de interesses.

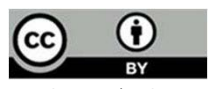

Copyright (C2021

\section{RESUMO}

Este trabalho visou evidenciar a importância da detecção precoce da coroidite interna punctata e destacar sua fisiopatologia inflamatória e possíveis diagnósticos diferenciais dentro das white dot syndromes. $\bigcirc$ destaque foi dado principalmente à coroidite multifocal e à panuveíte, ao se demonstrar sua epidemiologia peculiar em mulheres jovens, caracterizar sua apresentação clínica típica na fundoscopia e explorar as vantagens e as desvantagens de realizar os exames complementares que fazem parte da análise multimodal útil para o diagnóstico (especialmente a angiografia fluoresceínica, a tomografia de coerência óptica e a indocianina verde). Descreve-se o caso de uma mulher de 28 anos diagnosticada com coroidite interna punctata com membrana neovascular coroidal em olho direito. O tratamento foi realizado com injeção intravítrea de aflibercepte e corticoterapia sistêmica $1 \mathrm{mg} / \mathrm{kg}$ ao dia. Este relato é importante por permitir debater o manejo da coroidite interna punctata durante a gestação e a decisão de realizar o tratamento mediante uma diversidade de opções terapêuticas.

\begin{abstract}
This work aimed to demonstrate the importance of early detection of punctate inner choroidopathy, highlighting the pathophysiology of inflammation and the differential diagnoses among white dot syndromes. Special attention was given to multifocal choroiditis and panuveitis, by demonstrating the peculiar epidemiology in young women, characterizing the typical clinical presentation in ophthalmoscopy, and exploring the advantages and disadvantages of performing the complementary examinations, which are part of the multimodal analysis useful for diagnosis (particularly fluorescein angiography, optical coherence tomography, and indocyanine green). We report the case of a 28-year-old female, diagnosed as punctate inner choroidopathy with choroidal [N.T. no título aparece subretinal = subrretiniana] neovascular membrane in the right eye. She was treated with intravitreal injection of aflibercept and systemic corticosteroid $1 \mathrm{mg} / \mathrm{kg} /$ day. This case report is important for addressing the management of punctate inner choroidopathy during pregnancy, and the decision to carry out treatment considering diverse therapeutic options.
\end{abstract}




\section{INTRODUÇÃO}

A coroidite interna punctata (PIC) é uma uveíte posterior pertencente ao grupo das síndromes dos pontos brancos (WDS, do inglês white dot syndromes). ${ }^{(1)}$ Ocorre frequentemente em mulheres com média de idade de 32 anos (variação de 16 a 64 anos), com miopia, evoluindo com cicatrizes coriorretinianas e, eventualmente, formação de membrana neovascular coroidal (MNVC), também chamada de membrana neovascular sub-retiniana, ou até fibrose sub-retiniana, podendo resultar em perda visual ou diminuição da acuidade visual (AV) permanente. A tomografia de coerência óptica (OCT) e a angiografia fluoresceínica (AF) são utilizadas para identificar e monitorar a MNVC em pacientes com PIC. ${ }^{(1)}$ As modalidades terapêuticas para MNVC relacionada à PIC não estão bem estabelecidas, mas os regimes de tratamento relatados incluem fotocoagulação a laser, tratamento fotodinâmico e injeções intravítreas de antifator de crescimento endotelial vascular (VEGF). ${ }^{(2,3)}$ As abordagens terapêuticas para PIC são feitas com esteroides sistêmicos, tópicos e perioculares, além de agentes imunomoduladores poupadores de corticoide.

\section{RELATO DO CASO}

Mulher, 28 anos, caucasiana, procurou atendimento com baixa AV há 3 anos. Ao exame oftalmológico, apresentou AV com a melhor correção igual 20/80 no olho direito (OD) e 20/20 no olho esquerdo (OE). A refração dinâmica evidenciou miopia de -1,00DE em OD e -0,75DE -050DC a 60은 em OE. A biomicroscopia e a tonometria foram normais. A fundoscopia revelou cicatrizes coroidais arredondadas no polo posterior em ambos os olhos (AO) e MNVC com hemorragia adjacente no OD (Figura 1). A autofluorescência de fundo (FAF, fundus autofluorescence) demonstrou lesões hipoautofluorescentes em AO (Figura 2).

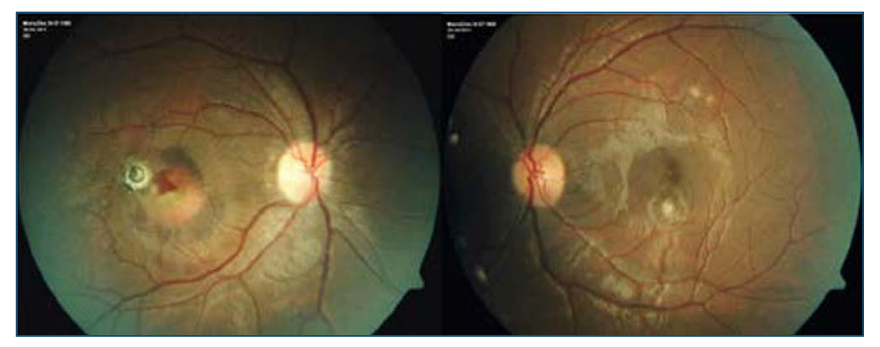

Figura 1. Retinografia revela cicatrizes coroidais arredondadas no polo posterior em ambos os olhos e membrana neovascular coroidal com hemorragia adjacente em olho direito.

Exames das funções renal e hepática, hemograma, coagulograma e rastreio infeccioso com teste tuberculínico, radiografia de tórax, VDRL, FTA-abs (sigla do inglês fluorescent treponemal antibody absorption test), imunoglobulina

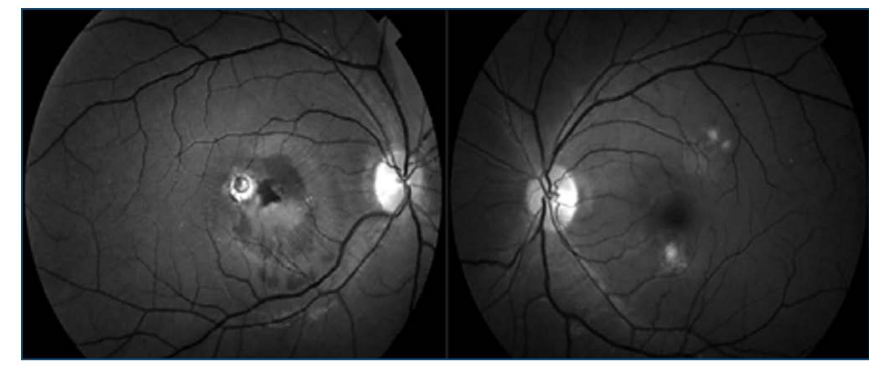

Figura 2. Autofluorescência revelou lesões hipoautofluorescentes em ambos os olhos.

G (IgG) e imunoglobulina M (IgM) para toxoplasmose, anti-HIV, anti-HBS, anti-HCV e HBsAg foram negativos.

No OD, a AF demonstrou hiperfluorescência precoce no subepitélio pigmentar da retina com pooling e hipofluorescência por bloqueio e, no OE, hiperfluorescência em todas as fases relacionadas às cicatrizes coroidais (Figura 3). A OCT revelou, no OD, MNVC ativa, com aumento da espessura foveal, desorganização das camadas da retina, fluido sub-retiniano e edema cistoide adjacentes, aumento da espessura justafoveal, hiper-refletividade no epitélio pigmentar da retina (EPR) e fotorreceptores devido à cicatriz coroidal (Figura 4). O diagnóstico estabelecido foi PIC com MNVC em OD, tratada com injeção intravítrea de aflibercepte e corticoterapia sistêmica $1 \mathrm{mg} / \mathrm{kg}$ ao dia.

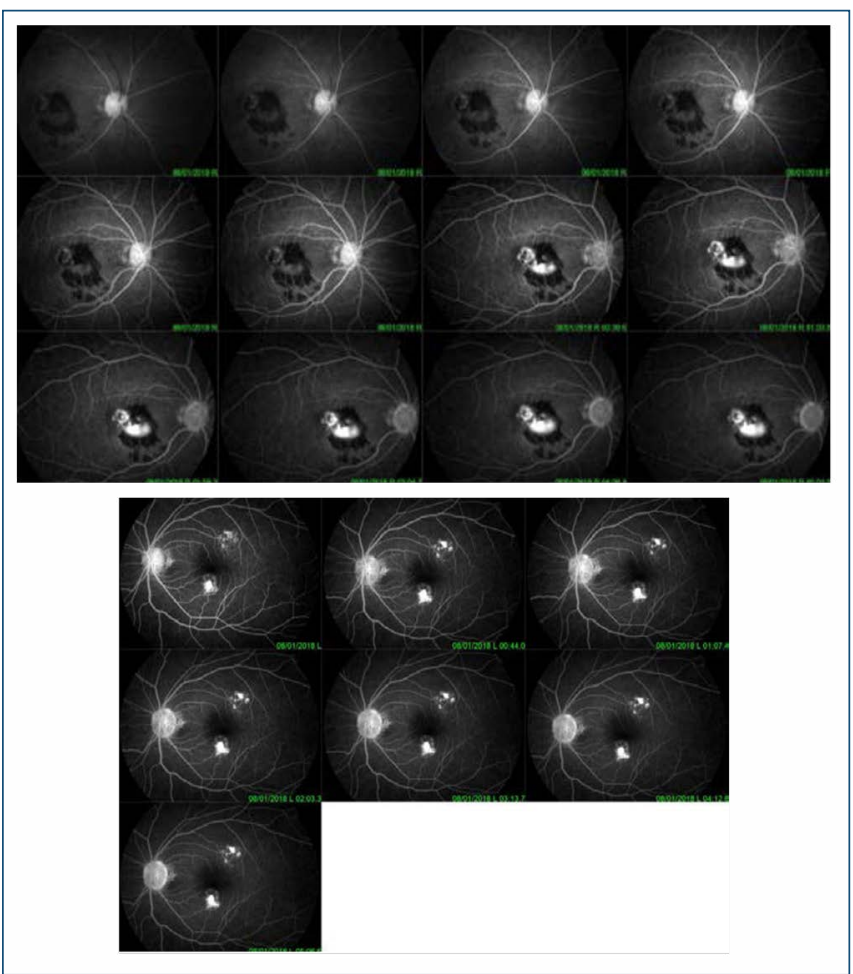

Figura 3. Angiografia fluoresceínica apresentou hiperfluorescência precoce em subepitélio pigmentar da retina com pooling em olho direito, hipofluorescência por bloqueio em olho esquerdo e hiperfluorescência em todas as fases relacionadas às cicatrizes coroidais. 


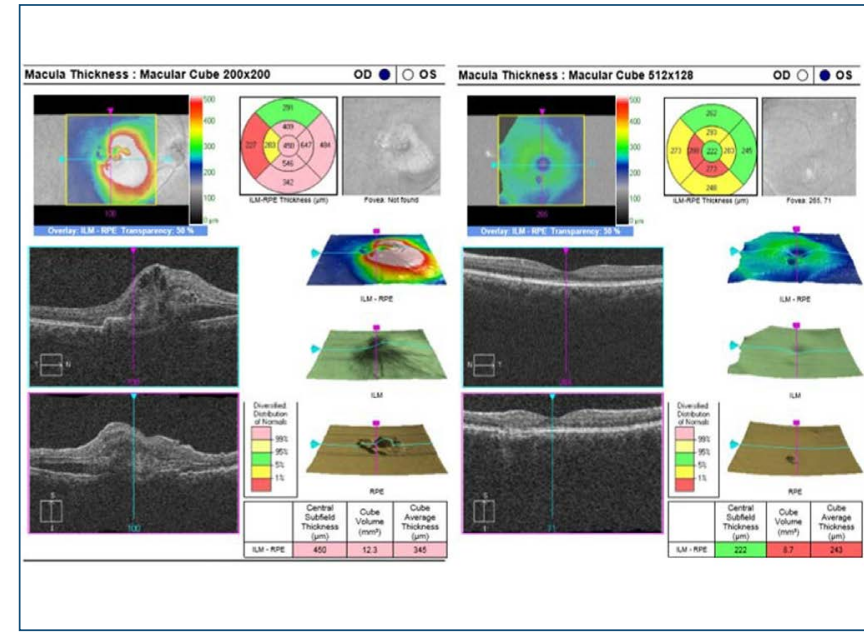

Figura 4. Tomografia de coerência óptica pré-injeção intravítrea demonstrou membrana neovascular coroidal ativa com aumento da espessura foveal, desorganização das camadas da retina, fluido sub-retiniano e edema cistoide adjacentes em olho direito, além de aumento da espessura justafoveal, hiper-refletividade no epitélio pigmentar da retina e fotorreceptores, devido à cicatriz coroidal.

Após 7 dias do início do tratamento, houve melhora da AV com correção em OD (20/50). Foi realizada OCT após a injeção, que demonstrou redução parcial da MNVC (Figura 5B). Após 30 dias, foi realizada nova injeção em OD. O seguimento com OCT revelou lesão sem alterações evolutivas em OD e melhora anatômica completa em OE (Figura 6). A imunossupressão sistêmica como terapia poupadora de corticoide não foi realizada na ocasião prescrita, pois a paciente apresentava-se gestante. Após 7 meses, houve piora da AV, com correção igual a 20/160 e 20/50. Nova retinografia não demonstrou modificações aparentes (Figura 7), e a OCT revelou piora da MNVC em OD (Figura 8). A angiotomografia de coerência óptica (OCT-A) demonstrou alteração da coriocapilar em topografia da MNVC em OD (Figura 9).

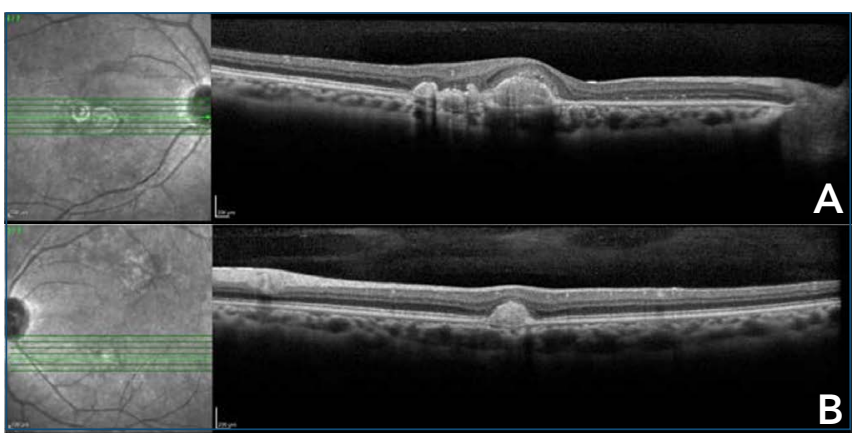

Figura 5. Tomografia de coerência óptica após primeira injeção intravítrea evidenciando redução parcial da membrana neovascular coroidal em olho direito. (A) Da esquerda para a direita: olho direito e esquerdo, respectivamente. (B) De cima para baixo: olho direito e esquerdo, respectivamente.

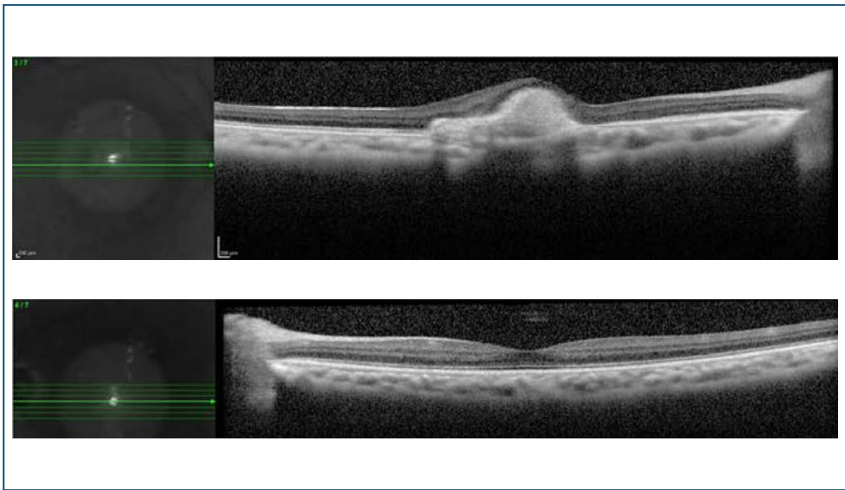

Figura 6. Tomografia de coerência óptica após segunda injeção intravítrea revelando lesão sem alterações evolutivas em olho direito e melhora completa em olho esquerdo.

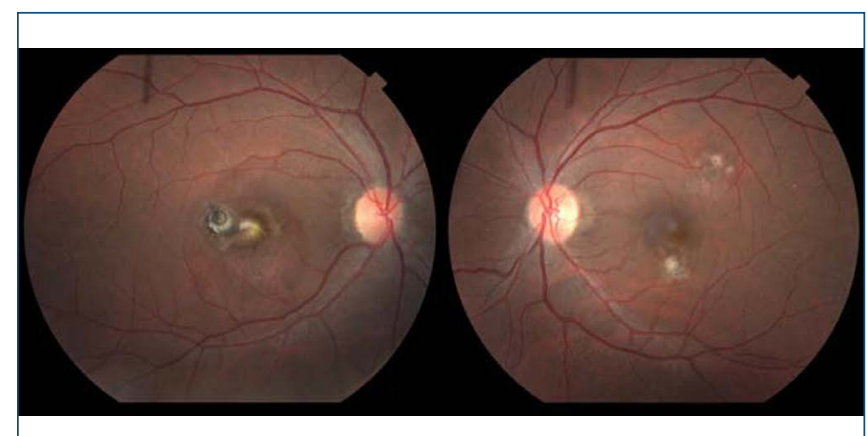

Figura 7. Retinografia após piora da acuidade visual sem alterações relevantes.

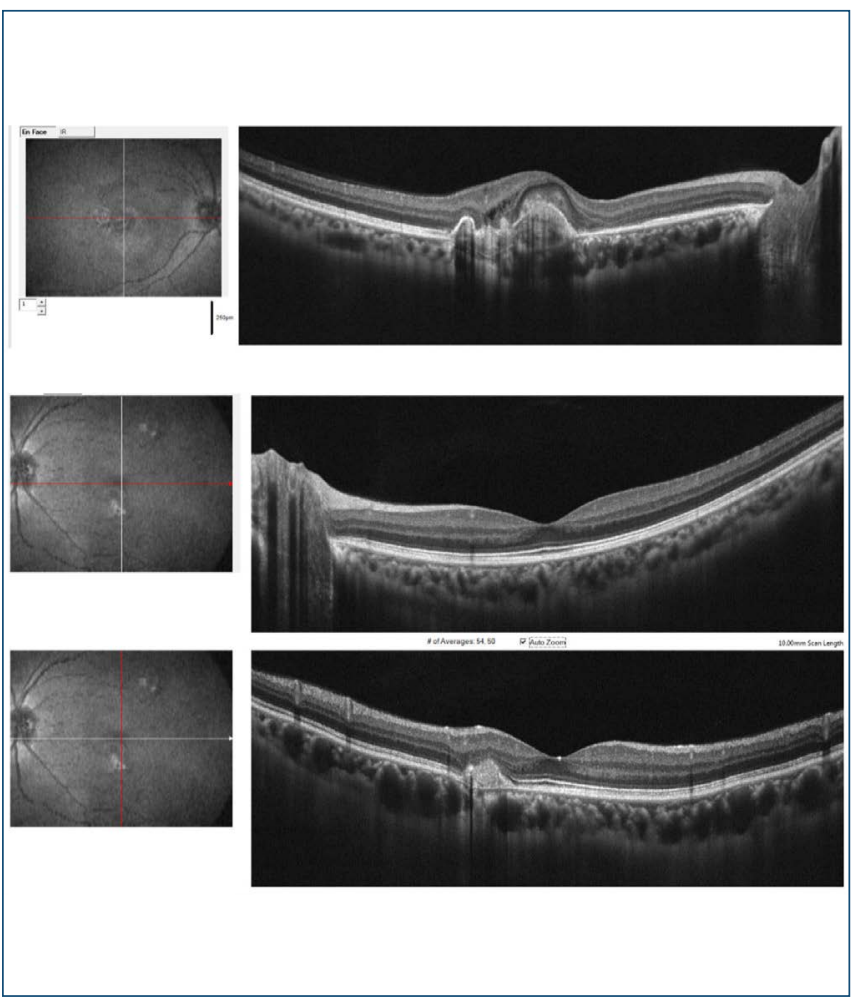

Figura 8. Tomografia de coerência óptica, após piora da acuidade visual, revelou agravamento da membrana neovascular coroidal em olho direito. 


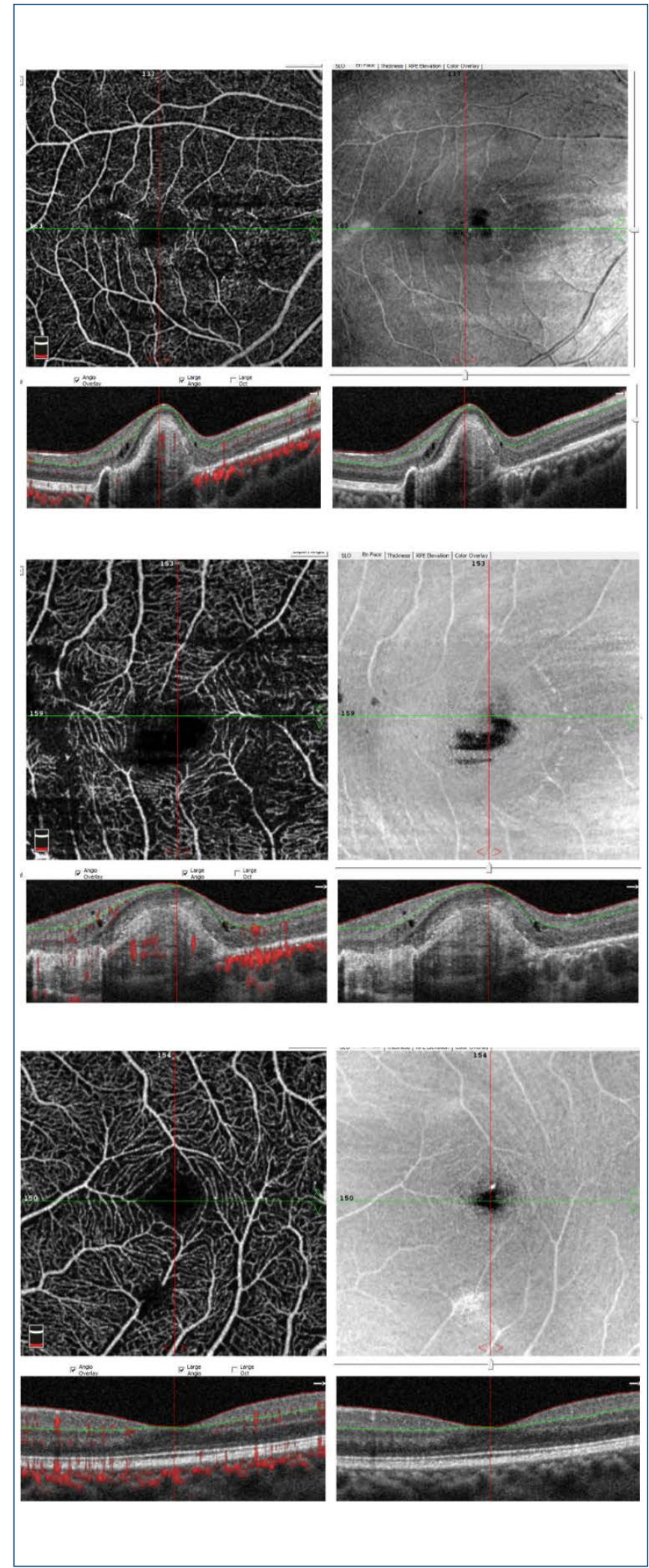

Figura 9. Angiotomografia de coerência óptica após piora da acuidade visual mostrou alteração da coriocapilar em topografia da membrana neovascular coroidal em olho direito. (A) Angiotomografia de coerência óptica do olho direito: corte transversal vertical. (B) Angiotomografia de coerência óptica do olho esquerdo: corte transversal horizontal. (C): Angiotomografia de coerência óptica do olho esquerdo: corte transversal horizontal.

\section{DISCUSSÃO}

A PIC tende a ocorrer em pacientes caucasianos, jovens, do sexo feminino (90\%) e saudáveis. Os principais sintomas são: fotopsias, turvação visual, escotomas paracentrais e metamorfopsia. ${ }^{(1)}$

É difícil fazer uma estimativa precisa da incidência e da prevalência de PIC, principalmente porque são amplas sua apresentação e sua gravidade, de modo que muitos casos podem permanecer desconhecidos. Além disso, sua classificação é incerta: é uma entidade distinta da coroidite multifocal com panuveíte (CMFP) ou trata-se de parte do mesmo espectro? Sugere-se que a PIC e a coroidite multifocal com panuveíte representem um espectro único da doença, que é modificado por fatores do hospedeiro, incluindo sua imunorregulação e causa a variedade de fenótipos clínicos observados. ${ }^{(4,5)}$

A fisiopatologia da PIC ainda é controversa, podendo se tratar de uma doença inflamatória independente ou fazer parte de um espectro das WDS. A AV pode ser afetada ou não, dependendo da localização das lesões inflamatórias ou de suas complicações, e sua medida pode variar de 20/50, ou melhor, até 20/500, ou pior. O exame fundoscópico durante a fase aguda da doença mostra lesões bilaterais, discretas, pequenas $(100 \mu \mathrm{m}$ a $300 \mu \mathrm{m})$, bem delineadas, branco-amareladas ou acinzentadas, no polo posterior ao nível do EPR, coroide interna ou coriocapilar, que podem se tornar cicatrizes coriorretinianas branco-amareladas, com hiperpigmentação ao longo do tempo, poupando a região papilar. Essas lesões ocorrem na ausência de sinais de inflamação intraocular e também sem sinais de uveíte anterior ou vitreíte.(5) Neste caso, a paciente apresentou pior AV no OD, pois era uma lesão ocular justafoveal em atividade; no OE, entretanto, a AV foi menos afetada, por se tratar de lesão ocular cicatricial parafoveal.

As investigações utilizadas no diagnóstico e no monitoramento da PIC incluem principalmente a AF, a angiografia por indocianina verde (IGA) e a OCT, embora modalidades como FAF e OCT-A também sejam úteis. ${ }^{(5)}$ Os achados da IGA revelam acometimento da coriocapilar e a coroide interna com hipocianescência nas fases inicial, média e tardia, o que, provavelmente, corresponde à hipoperfusão da coroide. ${ }^{(4,6)}$

A OCT pode revelar elevação focal do EPR com espaço hiporrefletivo subjacente e atrofia focal da retina externa e EPR, além de pontos hiper-refletivos focais na coroide interna, com afinamento focal da coroide adjacente às lesões da PIC, permitindo detectar lesões subclínicas. Nesse caso, antes da terapia antiangiogênica, evidenciaram-se MNVC ativa por meio de aumento da espessura foveal, 
desorganização das camadas da retina, fluido sub-retiniano, material hiper-refletivo no subepitélio pigmentar da retina e intrarretiniano e edema cistoide adjacentes no OD. Após a terapia antiangiogênica, observou-se redução significativa da espessura foveal no OD, correspondendo à melhora da MNVC. Decorridos 4 meses da terapia citada, a OCT detectou recidiva da MNVC no OD, por meio de desorganização das estruturas retinianas e do aumento da espessura foveal e do edema intrarretiniano.

A AF revela lesões hiperfluorescentes precoces. ${ }^{(7)}$ No presente caso, a paciente apresentou lesões hiperfluorescentes precoces em $\mathrm{AO}$, defeito em janela no OD e hiperfluorescência tardia por vazamento de contraste.

A OCT-A pode servir como modalidade de imagem adjuvante útil no diagnóstico. ${ }^{(8,9)}$ Ela é capaz de detectar o fluxo sanguíneo anormal, correspondendo à atividade de membranas neovasculares de coroide, e diferenciá-lo de uma lesão inflamatória; demonstra vazios na coroide e no coriocapilar, que correspondem a áreas de hipoperfusão ou não perfusão da coroide; não é invasiva e evita possíveis efeitos colaterais raros, como a anafilaxia do corante com fluoresceína. ${ }^{\left({ }^{2}\right)}$ Neste caso, a paciente pôde realizar OCT-A somente após apresentar piora da AV, decorridos 4 meses de terapia antiangiogênica. $\mathrm{O}$ exame apresentou área de hipoperfusão na coriocapilar justafoveal e vascularização anormal no OD, correspondente à MNVC recidivada.

A AF e a OCT correspondem ao atual padrão-ouro para identificar e monitorar a MNVC. Ambas revelam o líquido sub-retiniano, destacando, portanto, o componente inflamatório da lesão. Entretanto, somente a OCT-A é capaz de revelar o fluxo anormal presente na lesão e destacar o componente vascular corresponde à MNVC.

A MNVC e a fibrose subretiniana são as complicações mais comuns e mais graves ameaçadoras da visão na PIC. ${ }^{(3,4)}$ Estudos mostraram que o VEGF desempenha papel essencial no desenvolvimento da MNVC. ${ }^{(10)} \mathrm{A}$ formação de MNVC parece ser desencadeada por reação inflamatória inicial ao nível da coriocapilar, o que resulta em um defeito focal de coroide, membrana de Bruch e EPR, ${ }^{(11,12)} \mathrm{com}$ alto risco de evolução para MNVC. ${ }^{(13)}$ Neste caso, a paciente apresentou MNVC do tipo 2, cuja rede neovascular cresce a partir da vasculatura coroide e atravessa o complexo de membrana do EPR-Bruch para o espaço sub-retiniano.

Atualmente, existe consenso sobre as lesões que devem receber tratamento oftalmológico imediato: lesões inflamatórias novas ou ativas, principalmente as que ameaçam a fóvea. ${ }^{(1)}$ No presente caso, a paciente foi tratada em OD pelo fato de a lesão ocular ser justafoveal. São úteis esteroides sistêmicos ou perioculares/ intraoculares na PIC e na MNVC associada à PIC. Terapia imunossupressora com esteroides sistêmicos ou outros imunomoduladores podem contribuir para controlar o gatilho inflamatório, diminuindo a frequência de ataques na PIC recorrente. A indicação de tratamento antiangiogênico intravítreo para MNVC é eficaz no tratamento da MNVC secundária à PIC. ${ }^{(14,15)} \mathrm{A}$ combinação de esteroides sistêmicos e tratamento antiangiogênico intravítreo pode ser opção interessante para abordar os componentes vasculares (angiogênicos) e extravasculares (inflamatórios) do crescimento da MNVC, como realizado no presente relato. ${ }^{(16)}$

É muito importante diferenciar CMFP de PIC, porque a abordagem terapêutica é significativamente diferente. A PIC foi descrita como múltiplas pequenas lesões $(100 \mu \mathrm{m}$ a $300 \mu \mathrm{m})$ no polo posterior, na ausência de células vítreas, podendo existir cistos intrarretinianos como sinal de sua restauração. Na CMFP, há múltiplas lesões, o tamanho da lesão é superior a $300 \mu \mathrm{m}$, e células vítreas estão presentes. ${ }^{(17,18)} \mathrm{A}$ diferença clínica entre elas é a presença de vitreíte e/ou irite em pacientes com CMFP. ${ }^{(3)} \mathrm{A}$ abordagem terapêutica na PIC geralmente é reservada para MNVC, quando se usa injeção intravítrea de anti-VEGF, mas podem ser considerados esteroides para lesões foveais. Na CMFP, geralmente a terapia com esteroides sistêmicos ou locais é a escolha para o quadro inflamatório, e a injeção intravítrea de anti-VEGF vem se mostrando opção eficaz no tratamento da neovascularização coroidal e do edema macular. ${ }^{(19)}$

A gravidez tem sido associada ao desenvolvimento ou à recorrência de MNVC. É provável que isso ocorra devido aos níveis elevados de fatores angiogênicos, como VEGF e fator de crescimento placentário. ${ }^{(5)}$ De fato, neste caso, observou-se que, na OCT, não houve alteração exuberante da MNVC, embora tenha ocorrido piora da AV durante a gestação. Mesmo sendo a OCT um exame não invasivo e que não coloca em risco a gestante e nem o feto, e, ainda, sabendo-se ser a lesão justafoveal um potencial ameaçador da visão, não foi possível realizar exames periódicos para detecção da recidiva de MNVC, como AV e OCT, pois houve pouca adesão da paciente, por residir em localidade distante do local de seu tratamento. Ademais, o uso do anti-VEGF durante a gestação pode causar efeitos colaterais sistêmicos na mãe e danos fetais. Isso porque a angiogênese desempenha papel importante no desenvolvimento da placenta e do feto. Acredita-se que ele pode ser administrado durante a gravidez somente quando o benefício potencial à mulher justifica os riscos potenciais ao feto. ${ }^{(20,21)}$ 
O prognóstico da PIC depende da localização das lesões e das complicações associadas, sendo a maioria dos casos autolimitada e com bom prognóstico visual. Cistos intrarretinianos, comuns na PIC, podem ser sinais de recuperação da doença. No entanto, a MNVC secundária à PIC pode levar à perda visual irreversível. ${ }^{(15,18)}$

No presente caso, pôde-se observar que o diagnóstico de PIC e a detecção precoce de sua complicação, como a MNVC no OD, por meio da OCT-A, viabilizaram a escolha da terapia mais adequada. O uso de antiangiogênico intravítreo inicialmente resultou em melhora da AV no OD e no controle da atividade da MNVC.

No entanto, o tratamento incompleto, devido à impossibilidade de a paciente iniciar terapia imunossupressora poupadora de corticoide em seu período gestacional concomitantemente ao curso da doença e ao aumento da produção de fatores angiogênicos inerentes a essa fase, pode ter contribuído para a piora evolutiva da AV, bem como da MNVC.

\section{REFERÊNCIAS}

1. Hampton BM, Aderman CM, Flynn HW Jr, Sridhar J. Optical coherence tomography angiography of punctate inner choroidopathy. Case Rep Ophthalmol Med. 2017;2017:4754231.

2. Tsaousis KT, Nassr M, Kapoor B, Konidaris VE, Tyradellis S, Empeslidis T. Long-term results of intravitreal bevacizumab and dexamethasone for the treatment of punctate inner choroidopathy associated with choroidal neovascularization: A case series. SAGE Open Med Case Rep. 2018;6:X18772478

3. Sá-Cardoso M, Dias-Santos A, Nogueira N, Nascimento H, BelfortMattos R. Punctate inner choroidopathy. Case Rep Ophthalmol Med. 2015;2015:371817.

4. Campos J, Campos A, Mendes S, Neves A, Beselga D, Sousa JC. Punctate inner choroidopathy: a systematic review. Med Hypothesis Discov Innov Ophthalmol. 2014;3(3):76-82.

5. Ahnood D, Madhusudhan S, Tsaloumas MD, Waheed NK, Keane PA, Denniston AK. Punctate inner choroidopathy: A review. Surv Ophthalmol. 2017;62(2):113-26.

6. Riaz KM, Jampol LM, Mirza RG. Fundus autofluorescence imaging in punctate inner choroidopathy with blind spot enlargement. Ocul Immunol Inflamm. 2012;20(6):460-3.
7. Shakoor A, Vitale AT. Imaging in the diagnosis and management of multifocal choroiditis and punctate inner choroidopathy. Int Ophthalmol Clin. 2012;52(4):243-56.

8. Kim EL, Thanos A, Yonekawa Y, Todorich B, Wolfe J, Randhawa S, et al Optical coherence tomography angiography findings in punctate inner choroidopathy. Ophthalmic Surg Lasers Imaging Retina. 2017;48(10):786-92.

9. Pohlmann D, Pleyer U, Joussen AM, Winterhalter S. Optical coherence tomography angiography in comparison with other multimodal imaging techniques in punctate inner choroidopathy. $\mathrm{Br} \mathrm{J}$ Ophthalmol. 2019;103(1):60-6.

10. Raven ML, Ringeisen AL, Yonekawa Y, Stem MS, Faia LJ, Gottlieb JL. Multimodal imaging and anatomic classification of the white dot syndromes. Int J Retina Vitreous. 2017;3(1):12.

11. Barth T, Zeman F, Helbig H, Gamulescu MA. Tratamento anti-VEGF intravítreo para neovascularização coroide secundária a pontilhada coroidopatia interna. Int Ophthalmol. 2017;38(3):923-31.

12. Agarwal A, Invernizzi A, Singh RB, Foulsham W, Aggarwal K, Handa S, et al. An update on inflammatory choroidal neovascularization: epidemiology, multimodal imaging, and management. J Ophthalmic Inflamm Infect. 2018;8(1):13.

13. Dolz-Marco R, Fine HF, Freund KB. How to differentiate myopic choroidal neovascularization, idiopathic multifocal choroiditis, and punctate inner choroidopathy using clinical and multimodal imaging findings. Ophthalmic Surg Lasers Imaging Retina. 2017;48(3):196-201.

14. Pohlmann D, Pleyer U, Joussen AM, Winterhalter S. Immunosuppressants and/or antivascular endothelial growth factor inhibitors in punctate inner choroidopathy? Follow-up results with optical coherence tomography angiography. Br J Ophthalmol. 2019;103(8):1152-7.

15. Peng Y, Zhang X, Mi L, Liu B, Zuo C, Li M, et al. Efficacy and safety of conbercept as a primary treatment for choroidal neovascularization secondary to punctate inner choroidopathy. BMC Ophthalmol. 2017;17(1):87.

16. Wu W, Li S, Xu H, Liu Y, Wang Y, Lai TY, et al. Treatment of punctate inner choroidopathy with choroidal neovascularization using corticosteroid and intravitreal ranibizumab. BioMed Res Int. 2018;2018:1585803.

17. Chen SN, Chen YL, Yang BC. Long-term outcome of punctate inner choroidopathy or multifocal choroiditis with active choroidal neovascularization managed with intravitreal bevacizumab. Ocul Immunol Inflamm. 2020;28(1):33-8.

18. Gan Y, Zhang X, Chen L, Wen F. Intraretinal cystoid spaces in regression of punctate inner choroidopathy lesions. Ocul Immunol Inflamm. 2019;2019:1-9.

19. Niederer RL, Gilbert R, Lightman SL, Tomkins-Netzer O. Risk factors for developing choroidal neovascular membrane and visual loss in punctate inner choroidopathy. Ophthalmology. 2018;125(2):288-94

20. Petri AS, Boysen K, Cehofski LJ, van Dijk EH, Dysli C, Fuchs J, et al Intravitreal injections with vascular endothelial growth factor inhibitors: a practical approach. Ophthalmol Ther. 2020;9(1):191-203

21. Polizzi S, Mahajan VB. Intravitreal anti-VEGF injections in pregnancy: case series and review of literature. J Ocul Pharmacol Ther. 2015;31(10):605-10. 